\title{
Damage Localization for Structural Health Monitoring Using Retrospective Cost Model Refinement
}

\author{
Anthony M. D’Amato, ${ }^{*}$ Amy R. Wu, ${ }^{\dagger}$ Kenny S. Mitchell, ${ }^{\ddagger}$ \\ University of Michigan, Ann Arbor, Michigan, 48109, United States of America \\ Sunil. L. Kukreja $\S$ \\ NASA Dryden Flight Research Center, Edwards, California, 93523, United States of America \\ Dennis S. Bernstein ฯ \\ University of Michigan, Ann Arbor, Michigan, 48109, United States of America
}

\begin{abstract}
Model refinement is directly applicable to health monitoring, where the goal is to determine changes in a system that may reflect damage. As data become available, an initial model for the undamaged system is updated; the updated model is then compared with the original model, and changes in the model are analyzed to deduce potential damage. Our goal is to use data to recursively refine a model that is physically representative of a structure. Specifically, we refine an initial model by first estimating the internal state of the physical system using an adaptive feedback control structure, and then using these state estimates we apply the same control structure to identify parameter updates to correct the initial model. In the case of a physically motivated model, updating these parameters allows system changes, such as damage to a system, to be localized. We demonstrate the method on several numerical and experimental problems.
\end{abstract}

\section{Nomenclature}

$G \quad$ Physical system

$G_{0} \quad$ Initial model of physical system

$\Delta_{x} \quad$ Dynamic map correction model

$\Delta_{w} \quad$ Input map correction model

$w \quad$ Measured input of physical system

$y \quad$ Measured output of physical system

$x \quad$ Internal state of physical system

$y_{\Delta} \quad$ Simulated output of state observer system

$x_{\Delta} \quad$ Simulated internal state of observer system

$\hat{y} \quad$ Simulated output of parameter update model

\section{Introduction}

Structural health monitoring (SHM) techniques are used to detect and diagnose damage in civil and aerospace structures. Among the many applications of these techniques, commercial and government organizations are interested in maintaining aging aircraft. In addition, deterioration can occur in satellites due to exposure to extreme temperatures, cosmic radiation, atomic oxygen, and impacts with foreign objects.

\footnotetext{
*NASA GSRP Fellow, Department of Aerospace Engineering, 1320 Beal, and AIAA student member.

${ }^{\dagger}$ Graduate Student, Department of Aerospace Engineering, 1320 Beal, and AIAA student member.

$\ddagger$ Graduate Student, Department of Aerospace Engineering, 1320 Beal, and AIAA student member.

$\S$ Engineer, DFRC-RC, and AIAA member.

๑ Professor, Department of Aerospace Engineering, 1320 Beal Avenue, and AIAA member.
} 
Model refinement is directly applicable to health monitoring, where the goal is to determine changes in a system that may reflect damage. As data become available, an initial model for the undamaged system is recursively updated; the updated model is then compared with the original model, and changes in the model are analyzed to deduce possible damage. If the model is based on spatial discretization, then the updated model can facilitate damage localization. Model refinement is variously known as model correction, empirical correction, model calibration, model updating, or uncertainty reduction, and relevant literature includes. $^{1-11}$

To perform model refinement, we assume that we are given an initial model consisting of known and unknown components, and the goal is to use data to refine (that is, correct, update, or modify) the unknown components. This approach can be viewed as a special case of system identification, where the goal is to identify a subsystem of a larger overall system.

This approach that we use for model refinement exploits the fact that the model refinement problem has the same architecture as adaptive control. In particular, the controller that is updated in adaptive control plays the role of the unknown subsystem, where the optimization process serves to update the controller (that is, the unknown subsystem) in order to minimize the control performance (that is, the modeling error). This relationship shows that adaptive control and model refinement are deeply related problems in system theory.

The approach that we adopt for model refinement is based on retrospective-cost-optimization (RCO) adaptive control. ${ }^{13}$ This approach requires minimal modeling of the plant, operates directly on sampled data, and is applicable to plants that are MIMO, unstable, and nonminimum phase. Model refinement based on retrospective-cost adaptive control has been demonstrated in. ${ }^{10}$

In RCO model refinement, the known subsystem serves as an initial model that includes components that are uncertain. In this way, model refinement updates the uncertain subsystem. For example, the initial model may represent a nominal (undamaged) condition, while the unknown subsystem may represent a perturbation due to damage. By using data to improve the accuracy of the unknown subsystem, model refinement can identify the damage state. Because of its adaptive control basis, RCO model refinement updates the unknown subsystem recursively as data become available. The method can thus be used either on-line or off-line.

In the present paper we go beyond previous work by using model refinement for damage localization. To do this, our goal is to identify specific parameters in the system that have a physical interpretation, such as damping and stiffness. The ability to identify specific physical parameters facilitates comparison with those parameters under nominal conditions, thus providing the means to localize and quantify the damage. Since the location of the damage is often not known a priori, RCO model refinement can be used with a collection of hypothesized damage scenarios, and the resulting performance of the algorithm can serve as a guide to determine the most likely damage in terms of both location and magnitude.

The approach to model refinement that we adopt in the present paper is an extension of the technique used in. ${ }^{10,11}$ This extension is necessitated by several factors, including the fact that RCO model reduction uses sampled data, whereas physical parameters reside within a continuous-time model. The extension that we develop in the present paper provides improved accuracy of physical parameters by using estimates of internal states of the physical system. Standard estimation techniques such as the Kalman filter cannot be used for this purpose due to uncertainty in the model of the physical system. Alternatively, we adopt a two-stage approach. First, we perform RCO model refinement using a high-order model of the unknown subsystem. Although this step provides a model of the unknown subsystem that is not consistent with the unknown constant parameters, it has the advantage of providing a realistic estimate of the input to the unknown subsystem. In the second step, the estimated input to the unknown subsystem is used within RCO model refinement along with a constant model of the unknown parameters, that is, an unknown subsystem of zeroth order.

In the present paper we describe and demonstrate this two-stage approach to damage localization and quantification. In Section 2, we describe the general model refinement problem for parameter estimation. In Section 3, we describe a state estimation technique when the system model is not exactly known. Section 4 , describes parameter estimation. Sections 5, 6 and 7 demonstrate the method on numerical and experimental examples. 


\section{Problem Formulation}

Consider the SISO continuous-time state space system

$$
\begin{aligned}
\dot{\tilde{x}} & =\left(\tilde{A}_{\mathrm{c}}+\Delta \tilde{A}_{\mathrm{c}}\right) \tilde{x}+\left(\tilde{B}_{\mathrm{c}}+\Delta \tilde{B}_{\mathrm{c}}\right) w, \\
y & =(\tilde{C}+\Delta \tilde{C}) \tilde{x},
\end{aligned}
$$

where $\tilde{A}_{\mathrm{c}}, \Delta \tilde{A}_{\mathrm{c}} \in \mathbb{R}^{n \times n}, \tilde{B}_{\mathrm{c}}, \Delta \tilde{B}_{\mathrm{c}} \in \mathbb{R}^{n}, \tilde{C}$, and $\Delta \tilde{C} \in \mathbb{R}^{1 \times n}$. Furthermore, $\Delta \tilde{A}_{\mathrm{c}}, \Delta \tilde{B}_{\mathrm{c}}, \Delta \tilde{C}$ represent uncertainty in the system model. The system (1), (2) can be transformed such that all uncertainty appears in the dynamic and input map matrices. We write the transfer function

$$
G(s)=(C+\Delta C)\left[s I-\left(A_{\mathrm{c}}+\Delta A_{\mathrm{c}}\right)\right]^{-1}\left(B_{\mathrm{c}}+\Delta B_{\mathrm{c}}\right)=\frac{1}{\alpha(s)+\alpha_{\Delta}(s)}\left(\beta(s)+\beta_{\Delta}(s)\right),
$$

where

$$
\begin{aligned}
\alpha(s) & \triangleq s^{n}+\alpha_{1} s^{n-1}+\cdots+\alpha_{n-1} s+\alpha_{n}, \\
\alpha_{\Delta}(s) & \triangleq s^{n}+\alpha_{\Delta, 1} s^{n-1}+\cdots+\alpha_{\Delta, n-1} s+\alpha_{\Delta, n}, \\
\beta(s) & \triangleq s^{n-d} \beta_{d}+s^{n-d-1} \beta_{d+1}+\ldots+s \beta_{n-1}+\beta_{n}, \\
\beta_{\Delta}(s) & \triangleq s^{n-d} \beta_{\Delta, d}+s^{n-d-1} \beta_{\Delta, d+1}+\ldots+s \beta_{\Delta, n-1}+\beta_{\Delta, n},
\end{aligned}
$$

where $d$ is the relative degree. From (4)-(7) we transform the realization of (1), (2) to the observable canonical form

$$
\begin{aligned}
& \dot{x}=\left(A_{\mathrm{c}}+\Delta A_{\mathrm{c}}\right) x+\left(B_{\mathrm{c}}+\Delta B_{\mathrm{c}}\right) w, \\
& y=C x,
\end{aligned}
$$

where

$$
\begin{aligned}
A_{\mathrm{c}}+\Delta A_{\mathrm{c}} & =\left[\begin{array}{ccccc}
-\alpha_{1}-\alpha_{\Delta, 1} & 1 & 0 & \cdots & 0 \\
-\alpha_{2}-\alpha_{\Delta, 2} & 0 & \ddots & \ddots & \cdots \\
\vdots & \vdots & \ddots & \ddots & 0 \\
-\alpha_{n-1}-\alpha_{\Delta, n-1} & 0 & \cdots & 0 & 1 \\
-\alpha_{n}-\alpha_{\Delta, n} & 0 & \cdots & 0 & 0
\end{array}\right], \quad B_{\mathrm{c}}+\Delta B_{\mathrm{c}}=\left[\begin{array}{c}
\beta_{d}+\beta_{\Delta, d} \\
\vdots \\
\beta_{n}+\beta_{\Delta, n}
\end{array}\right], \\
C & =\left[\begin{array}{cc}
1 & 0_{1 \times n-1}
\end{array}\right] .
\end{aligned}
$$

Therefore all uncertainty appears in the dynamic and input map matrices; the output map is exactly known. The initial model $G_{0}$ is created from the known portion of the physical system

$$
\begin{aligned}
& \dot{x}=A_{\mathrm{c}} x+B_{\mathrm{c}} u, \\
& y=C x .
\end{aligned}
$$

Choosing $u=\Delta_{x} x+\Delta_{w} w$ we can write the unknown components in feedback with the known components, where $\Delta A_{\mathrm{c}}=B_{\mathrm{c}} \Delta_{x}$ and $\Delta B_{\mathrm{c}}=B_{\mathrm{c}}\left(I+\Delta_{w}\right)$. Figure 1 shows how the physical system is separated into a known initial model $G_{0}$ with unknown feedback $\left[\Delta_{x} \Delta_{w}\right]$. We note that the signal $u$ and the internal state $x$ are not accessible. Therefore, $\Delta_{x}$ and $\Delta_{w}$ cannot be directly identified. We obtain the closed-loop transfer function from $w$ to $y$,

$$
y=G w=G_{0, y}\left[\Delta_{x}\left[I-G_{0, x} \Delta_{x}\right]^{-1} G_{0, x}+I\right] \Delta_{w} w .
$$

The goal is to determine an estimate $\left[\hat{\Delta}_{x} \hat{\Delta}_{w}\right]$ of $\left[\Delta_{x} \Delta_{w}\right]$, where the refined model is the interconnection of $G_{0}$ and $\left[\hat{\Delta}_{x} \hat{\Delta}_{w}\right]$ such that the output of the refined model

$$
\hat{y}=G_{0, y}\left[\hat{\Delta}_{x}\left[I-G_{0, x} \hat{\Delta}_{x}\right]^{-1} G_{0, x}+I\right] \hat{\Delta}_{w} w,
$$




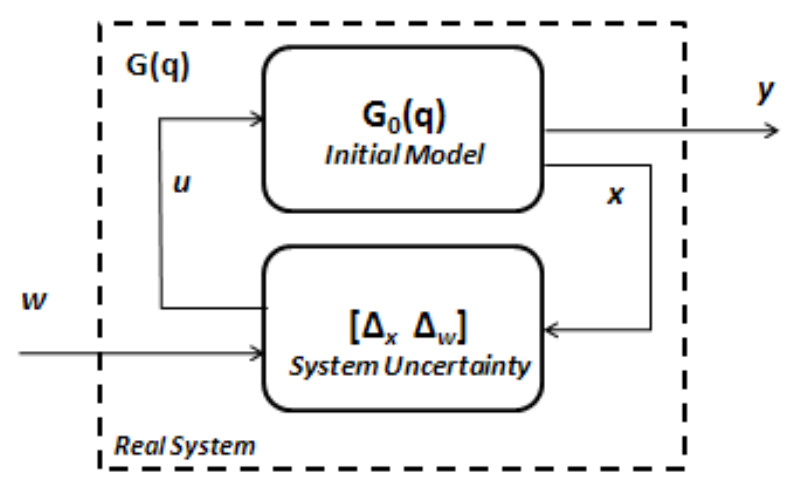

Figure 1. This block diagram represents how the real system can be separated into two system in feedback, where one system is the known initial model $G_{0}$, and $\left[\Delta_{x} \Delta_{w}\right]$ contains the uncertainty in the system.

approximates the physical system, in the sense that, $z_{\Delta}=y-\hat{y}$ is small.

To identify the feedback term $\left[\Delta_{x} \Delta_{w}\right]$ using the given initial model $G_{0}$, we use an adaptive feedback model structure. In order to determine a static estimate of $\left[\Delta_{x} \Delta_{w}\right]$, we require an estimate of the internal state of $G$. Therefore we propose a two step method in which we use the retrospective cost optimization ( $\mathrm{RCO}$ ) adaptive control algorithm ${ }^{13}$ to first obtain estimates $x_{\Delta}$ of $x$ and, second, we again use RCO with the state estimates from the first step to obtain an estimate $\left[\hat{\Delta}_{x} \hat{\Delta}_{w}\right]$, of $\left[\Delta_{x} \Delta_{w}\right]$. The only signals available to the controller are the measured input signal $w$, the measured output signal $y$, the simulated output $y_{\Delta}$, and the simulated output using the updated state estimated $\hat{y}$. Figure 2 demonstrates the problem architecture. It should be noted that the two steps are performed concurrently, and thus the method is used recursively, that is, the estimate $\left[\hat{\Delta}_{x} \hat{\Delta}_{w}\right]$ of $\left[\Delta_{x} \Delta_{w}\right]$ is updated as data become available.

\section{State Estimation with Model Uncertainty}

We require estimates of the internal state of the physical system. To do this, consider the observer

$$
\begin{aligned}
& \dot{x_{\mathrm{e}}}=A_{\mathrm{c}} x_{e}+B_{\mathrm{c}} u+L\left(y-y_{e}\right), \\
& y_{\mathrm{e}}=C x
\end{aligned}
$$

where $L \in \mathbb{R}^{n}, x_{\mathrm{e}} \in \mathbb{R}^{n}$ is an estimate of $x$, and $e \triangleq x-x_{e}$, then

$$
\dot{e}=\left(A_{\mathrm{c}}-L C\right) e+\Delta A_{\mathrm{c}} x+\Delta B_{\mathrm{c}} y .
$$

Choosing $L$ such that $A-L C$ is asymptotically stable does not guarantee a reduction in $e$ since $\Delta A_{\mathrm{c}}$ and $\Delta B_{\mathrm{c}}$ are unknown.

We propose to obtain estimates of the internal state $x$, of (8), (9) using model refinement, which is accomplished by using the RCO adaptive control algorithm. To implement RCO, which is a sampled-data time adaptive control method, consider (8), (9) discretized with the sampling interval $T_{\mathrm{s}}$, where

$$
\begin{aligned}
A+\Delta A & \left.=e^{\left(A_{\mathrm{c}}+\Delta A_{c}\right) T_{\mathrm{s}}}, \quad B+\Delta B=\left[A_{\mathrm{c}}+\Delta A_{c}\right]^{-1}\right)(A+\Delta A-I)\left(B_{c}+\Delta B_{c}\right), \\
A & =e^{A_{\mathrm{c}} T_{\mathrm{s}}}, \quad B=A_{\mathrm{c}}^{-1}[A-I] B_{c}
\end{aligned}
$$

and

$$
\begin{aligned}
x(k+1) & =(A+\Delta A) x(k)+(B+\Delta B) w(k), \\
y(k) & =C x(k) .
\end{aligned}
$$




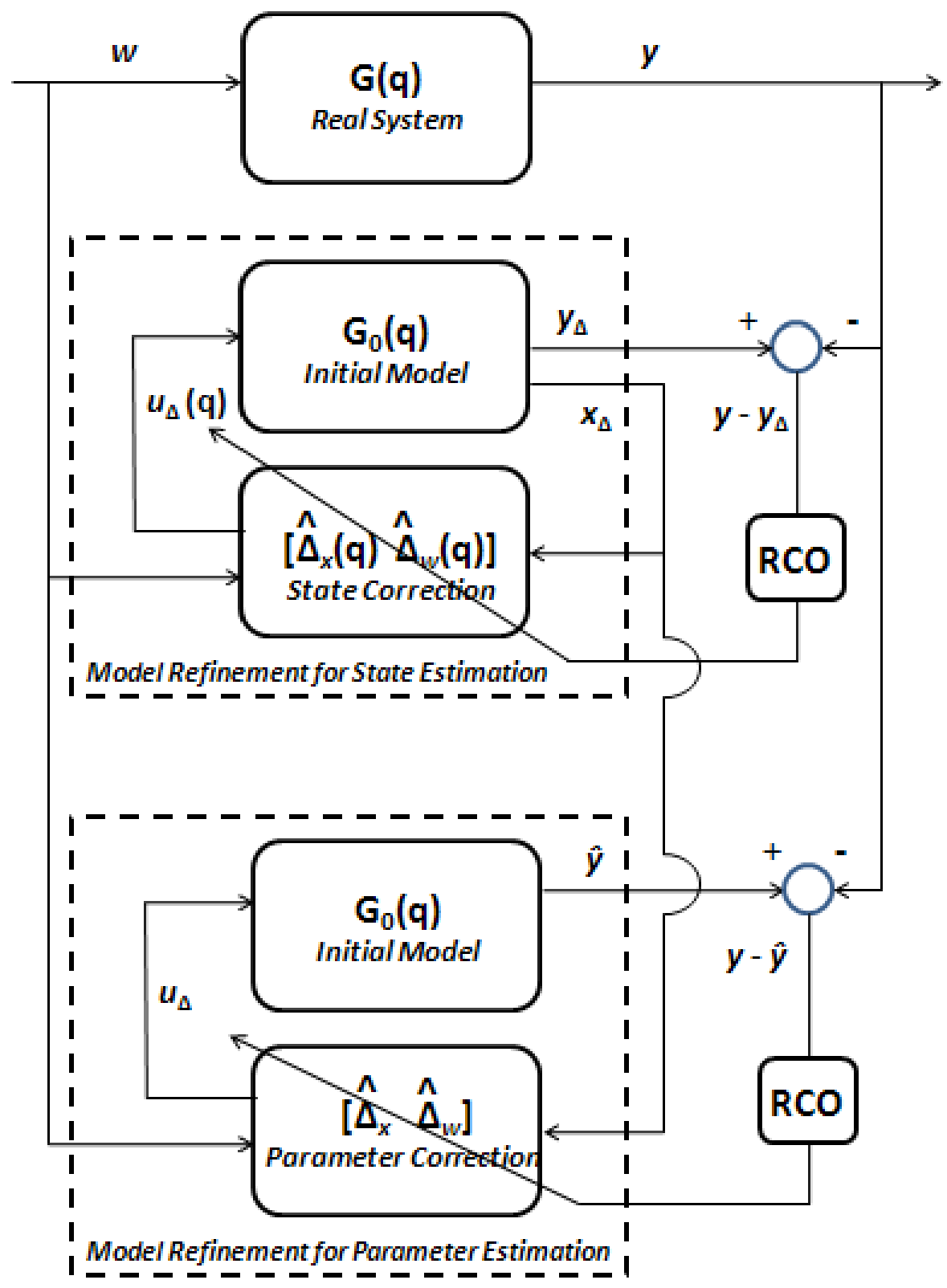

Figure 2. This block diagram represents the method for identifying $\left[\Delta_{x} \Delta_{w}\right]$, where estimates of the unknown internal state $x$ are determined by computing $u_{\Delta}(\mathbf{q})$. Using the estimate $x_{\Delta}$ of the internal state we obtain the estimate $\left[\hat{\Delta}_{x} \hat{\Delta}_{w}\right]$ of $\left[\Delta_{x} \Delta_{w}\right]$. Here, $\underline{\mathbf{q}}$ denotes the forward shift operator. 
We build a state estimator using the known system parameters. To do this, let $x_{\Delta}(k)$ be an estimate of $x(k)$ satisfying

$$
\begin{aligned}
x_{\Delta}(k+1) & =A x_{\Delta}(k)+B u_{\Delta}(k), \\
y_{\Delta}(k) & =C x_{\Delta}(k),
\end{aligned}
$$

where $u_{\Delta}(k)=\hat{\Delta}_{x}(\mathbf{q}, k) x_{\Delta}(k)+\hat{\Delta}_{w}(\mathbf{q}, k) w(k)$, where $\mathbf{q}$ is the forward shift operator and $\hat{\Delta}_{x}(\mathbf{q}, k)$ and $\hat{\Delta}_{w}(\mathbf{q}, k)$ are transfer function operators at step $k$. Then (23) becomes

$$
x_{\Delta}(k+1)=\left(A+B \hat{\Delta}_{x}(\mathbf{q}, k)\right) x_{\Delta}(k)+B \hat{\Delta}_{w}(\mathbf{q}, k) .
$$

$\hat{\Delta}_{x}(\mathbf{q}, k)$ and $\hat{\Delta}_{w}(\mathbf{q}, k)$ are determined using retrospective cost optimization.

\section{A. Retrospective Cost Optimization}

Retrospective cost optimization (RCO) depends on several parameters that are selected a priori. Specifically, $n_{\mathrm{c}}$ is the estimated plant order, $p \geq 1$ is the data window size, and $\mu$ is the number of Markov parameters obtained from the known model. The methodology for choosing these parameters is as follows. $n_{c}$ is overestimated, that is, chosen to be greater than the expected order of $\left[\Delta_{x} \Delta_{w}\right]$; for parameter estimation, the order of $\left[\Delta_{x} \Delta_{w}\right]$ is zero. $\mu$ is generally chosen to be 1 , however, a greater value may be necessary if nonminimum phase zeros are present in the initial model or suspected to be present in the unknown model.

The adaptive update law is based on a quadratic cost function, which involves a time-varying weighting parameter $\alpha(k)>0$, referred to as the learning rate since it affects the convergence speed of the adaptive control algorithm.

We use an exactly proper time-series controller of order $n_{\mathrm{c}}$ such that the control $u_{\Delta}(k)$ is given by

$$
u_{\Delta}(k)=\sum_{i=1}^{n_{\mathrm{c}}} M_{i}(k) u_{\Delta}(k-i)+\sum_{i=0}^{n_{\mathrm{c}}} N_{i}(k)\left[\begin{array}{c}
x_{\Delta}(k-i) \\
w(k-i)
\end{array}\right],
$$

where $M_{i} \in \mathbb{R}^{l_{u_{\Delta}} \times l_{u_{\Delta}}}, i=1, \ldots, n_{\mathrm{c}}$, and $N_{i} \in \mathbb{R}^{l_{u_{\Delta}} \times\left(l_{x}+l_{w}\right)}, i=0, \ldots, n_{\mathrm{c}}$, are given by an adaptive update law. The control can be expressed as

$$
u_{\Delta}(k)=\theta(k) \psi(k)
$$

where

$$
\theta(k) \triangleq\left[\begin{array}{llllll}
N_{0}(k) & \cdots & N_{n_{\mathrm{c}}}(k) & M_{1}(k) & \cdots & M_{n_{\mathrm{c}}}(k)
\end{array}\right]
$$

is the controller parameter block matrix and the regressor vector $\psi(k)$ is given by

$$
\psi(k) \triangleq\left[\begin{array}{c}
x_{\Delta}(k) \\
\vdots \\
x_{\Delta}\left(k-n_{\mathrm{c}}\right) \\
w(k) \\
\vdots \\
w\left(k-n_{\mathrm{c}}\right) \\
u_{\Delta}(k-1) \\
\vdots \\
u_{\Delta}\left(k-n_{\mathrm{c}}\right)
\end{array}\right] \in \mathbb{R}^{n_{\mathrm{c}} l_{u_{\Delta}}+\left(n_{\mathrm{c}}+1\right)\left(l_{x}+l_{w}\right)} .
$$

For positive integers $p$ and $\mu$, we define the extended performance vector $Z(k)$ and the extended control vector $U_{\Delta}(k)$ by

$$
Z(k) \triangleq\left[\begin{array}{c}
z(k) \\
\vdots \\
z(k-p+1)
\end{array}\right], \quad U_{\Delta}(k) \triangleq\left[\begin{array}{c}
w(k) \\
\vdots \\
w\left(k-p_{\mathrm{c}}+1\right)
\end{array}\right]
$$


where $p_{\mathrm{c}} \triangleq \mu+p$.

From (27), it follows that the extended control vector $U_{\Delta}(k)$ can be written as

$$
U_{\Delta}(k) \triangleq \sum_{i=1}^{p_{\mathrm{c}}} L_{i} \theta(k-i+1) \psi(k-i+1)
$$

where

$$
L_{i} \triangleq\left[\begin{array}{c}
0_{(i-1) l_{u_{\Delta}} \times l_{u_{\Delta}}} \\
I_{l_{u_{\Delta}}} \\
0_{\left(p_{c}-i\right) l_{u_{\Delta}} \times l_{u_{\Delta}}}
\end{array}\right] \in \mathbb{R}^{p_{c} l_{u_{\Delta}} \times l_{u_{\Delta}}}
$$

We define the surrogate performance vector $\hat{Z}(\hat{\theta}(k), k)$ by

$$
\hat{Z}(\hat{\theta}(k), k) \triangleq Z(k)-\bar{B}_{z w}(W(k)-\hat{W}(k)),
$$

where

$$
\hat{U}_{\Delta}(k) \triangleq \sum_{i=1}^{p_{c}} L_{i} \hat{\theta}(k) \psi(k-i+1)
$$

and $\hat{\theta}(k) \in \mathbb{R}^{l_{u_{\Delta}} \times\left[n_{c} l_{u_{\Delta}}+\left(n_{c}+1\right)\left(l_{w}+l_{x}\right)\right]}$ is the surrogate controller parameter block matrix. The block-Toeplitz surrogate control matrix $\bar{B}_{z u_{\Delta}}$ is given by

$$
\bar{B}_{z u_{\Delta}} \triangleq\left[\begin{array}{ccccccccc}
0_{l_{z} \times l_{u_{\Delta}}} & \cdots & 0_{l_{z} \times l_{u_{\Delta}}} & H_{d} & \cdots & H_{\mu} & 0_{l_{z} \times l_{u_{\Delta}}} & \cdots & 0_{l_{z} \times l_{u_{\Delta}}} \\
0_{l_{z} \times l_{u_{\Delta}}} & \ddots & \ddots & \ddots & \ddots & \ddots & \ddots & \ddots & \vdots \\
\vdots & \ddots & \ddots & \ddots & \ddots & \ddots & \ddots & 0_{z_{z} \times l_{u_{\Delta}}} & \vdots \\
0_{l_{z} \times l_{u_{\Delta}}} & \cdots & 0_{l_{z} \times l_{w}} & 0_{l_{z} \times l_{u_{\Delta}}} & \cdots & 0_{l_{z} \times l_{u_{\Delta}}} & H_{d} & \cdots & H_{\mu}
\end{array}\right]
$$

where the relative degree $d$ is the smallest positive integer $i$ such that the $i$ th Markov parameter $H_{i}$ of $\hat{\mathcal{L}}_{\mathrm{m}}$ is nonzero. The leading zeros in the first row of $\bar{B}_{z u_{\Delta}}$ account for the relative degree $d$. The algorithm places no constraints on either the value of $d>0$ or the rank of $H_{d}$ or $\bar{B}_{z u_{\Delta}}$. Furthermore, we define

$$
\begin{aligned}
& D(k) \triangleq \sum_{i=1}^{n_{\mathrm{c}}+\mu-1} \psi^{\mathrm{T}}(k-i+1) \otimes L_{i}, \\
& f(k) \triangleq Z(k)-\bar{B}_{z w} W(k) .
\end{aligned}
$$

We now consider the cost function

$$
J(\hat{\theta}, k) \triangleq \hat{Z}^{\mathrm{T}}(\hat{\theta}, k) R_{1}(k) \hat{Z}(\hat{\theta}, k)+\operatorname{tr}\left[R_{2}(k)(\hat{\theta}-\theta(k))^{\mathrm{T}} R_{3}(k)(\hat{\theta}-\theta(k))\right],
$$

where $R_{1}(k) \triangleq I_{p l_{z}}, R_{2}(k) \triangleq \alpha(k) I_{n_{c}\left(l_{w}+l_{v}\right)}$, and $R_{3}(k) \triangleq I_{l_{w}}$.

Substituting (31) and (32) into (36), $J$ is written as the quadratic form

$$
J(\hat{\theta}, k)=c(k)+b^{\mathrm{T}} \operatorname{vec} \hat{\theta}+(\operatorname{vec} \hat{\theta})^{\mathrm{T}} A(k) \operatorname{vec} \hat{\theta},
$$

where

$$
\begin{aligned}
A(k) & =D^{\mathrm{T}}(k) D(k)+\alpha(k) I, \\
b(k) & =2 D^{\mathrm{T}}(k) f(k)-2 \alpha(k) \operatorname{vec} \theta(k), \\
c(k) & =f(k)^{\mathrm{T}} R_{1}(k) f(k)+\operatorname{tr}\left[R_{2}(k) \theta^{\mathrm{T}}(k) R_{3}(k) \theta(k)\right] .
\end{aligned}
$$


Since $A(k)$ is positive definite, $J(\hat{\theta}, k)$ has the strict global minimizer

$$
\hat{\theta}(k)=\frac{1}{2} \operatorname{vec}^{-1}\left(A(k)^{-1} b(k)\right) .
$$

The controller gain update law is

$$
\theta(k+1)=\hat{\theta}(k) .
$$

Using the coefficients of the time series (26) we obtain $\hat{\Delta}_{x}(\mathbf{q}, k)$ and $\hat{\Delta}_{w}(\mathbf{q}, k)$.

\section{Parameter Estimation}

The transfer functions $\hat{\Delta}_{x}(\mathbf{q}, k)$ and $\hat{\Delta}_{w}(\mathbf{q}, k)$ are used to obtain the estimate $x_{\Delta}$, of $x$, that refines the initial model $G_{0}$. However, computing the closed loop

$$
\hat{y}=G_{0, y}\left[\hat{\Delta}_{x}(\mathbf{q}, k)\left[I-G_{0, x} \hat{\Delta}_{x}(\mathbf{q}, k)\right]^{-1} G_{0, x}+I\right] \hat{\Delta}_{w}(\mathbf{q}, k) w
$$

results in a model with more than $n$ states. For parameter estimation, the resultant closed-loop system must

contain the same number of states as the original system. Therefore we require a zeroth-order estimate of $\Delta_{x}$ and $\Delta_{w}$. Consider the refined model

$$
\begin{aligned}
\dot{\hat{x}}(k+1) & =\left(A+B \hat{\Delta}_{x}(k)\right) \hat{x}(k)+B \hat{\Delta}_{w} w(k), \\
\hat{y} & =C \hat{x},
\end{aligned}
$$

where we constrain the retrospective cost optimization to be $n_{c}=0$ and replace the state $\hat{x}$ at each step $k$ with the estimate of the internal state $x_{\Delta}$ obtained in the previous step. The static estimates $\left[\hat{\Delta}_{x} \hat{\Delta}_{w}\right]$ yield estimates of the unknown parameters. The idea is illustrated in Figure 2. To obtain the zeroth order transfer function we again implement RCO, with the following modifications. We use a static controller

$$
u_{\Delta}(k)=\theta(k)\left[\begin{array}{c}
x_{\Delta}(k) \\
w(k)
\end{array}\right],
$$

where $\theta(k) \in \mathbb{R}^{l_{w} \times\left(l_{x}+l w\right)}$. The control can be expressed as $u_{\Delta}(k)=\theta(k) \psi(k)$, where $\theta(k)$ is the new controller parameter block matrix and the new regressor vector $\psi(k)$ is

$$
\psi(k) \triangleq\left[\begin{array}{c}
x_{\Delta}(k) \\
\vdots \\
x_{\Delta}\left(k-n_{\mathrm{c}}\right) \\
w(k) \\
\vdots \\
w\left(k-n_{\mathrm{c}}\right)
\end{array}\right] \in \mathbb{R}^{l_{x}+l_{w}} .
$$

Using the zeroth order time-series controller with the new controller parameter block matrix and regressor, we implement RCO as in the previous section.

\section{A. Conversion to Continuous Time}

With (44)-(45), the discrete-time system can be converted to continuous time using the reverse of (20). Finally, changing the resultant realization to a canonical observable form, estimates of $\Delta A_{\mathrm{c}} \Delta B_{\mathrm{c}}$ are obtained.

\section{Single Degree of Freedom System}

For systems with a known model structure it is possible to estimate specific unknown parameters. Consequently, estimates of model parameters can be refined using data. To demonstrate this, consider the 


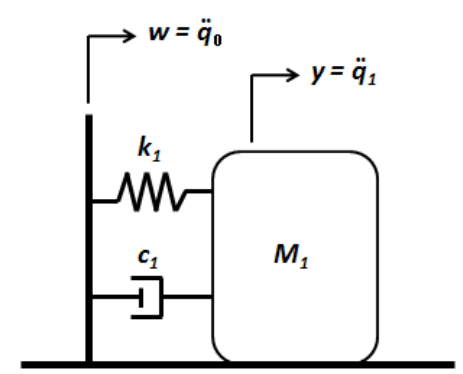

Figure 3. Mass-spring-damper 1-DOF structure.

mass-spring-damper structure shown in Figure 3. The goal is to estimate changes in stiffness and damping using only empirical data, namely, knowledge of the driving acceleration $w$, and output acceleration $y$. Note that, since $w$ and $y$ are accelerations, this mass-spring-damper system is a transmissibility.

The equations of motion for this system are

$$
M \ddot{x}+C_{\mathrm{d}} \dot{x}+K x=w .
$$

The state space form for this mass-spring-damper system is

$$
\tilde{A}_{\mathrm{c}}=\left[\begin{array}{cc}
0 & 1 \\
-M^{-1} K & -M^{-1} C_{\mathrm{d}}
\end{array}\right], \quad \tilde{B}_{\mathrm{c}}=\left[\begin{array}{ll}
0 & -1
\end{array}\right]^{\mathrm{T}}, \quad \tilde{C}=\left[\begin{array}{cc}
-M^{-1} K & -M^{-1} C_{\mathrm{d}}
\end{array}\right], \quad D=0 .
$$

We choose $M=200, K=63500, C_{\mathrm{d}}=15000$, which are the parameters of the initial model. Furthermore, we assume that the physical system has undergone a change with $M+\Delta M=803$ and $C_{\mathrm{d}}+\Delta C_{\mathrm{d}}=7000$.

We implement RCO to obtain estimates of the transfer functions $\hat{\Delta}_{x}(\mathbf{q}, k)$ and $\hat{\Delta}_{w}(\mathbf{q}, k)$ used to obtain $x_{\Delta}$. The RCO parameter selections are $n_{c}=30, p=100, \mu=1, a=1000$. These parameters are selected to yield the best performance in terms of minimization of $y_{\Delta}-y$. Generally, good performance can be obtained with a range of parameter selections. Figure 4 shows the history of the performance variable $y_{\Delta}-y$. Figure

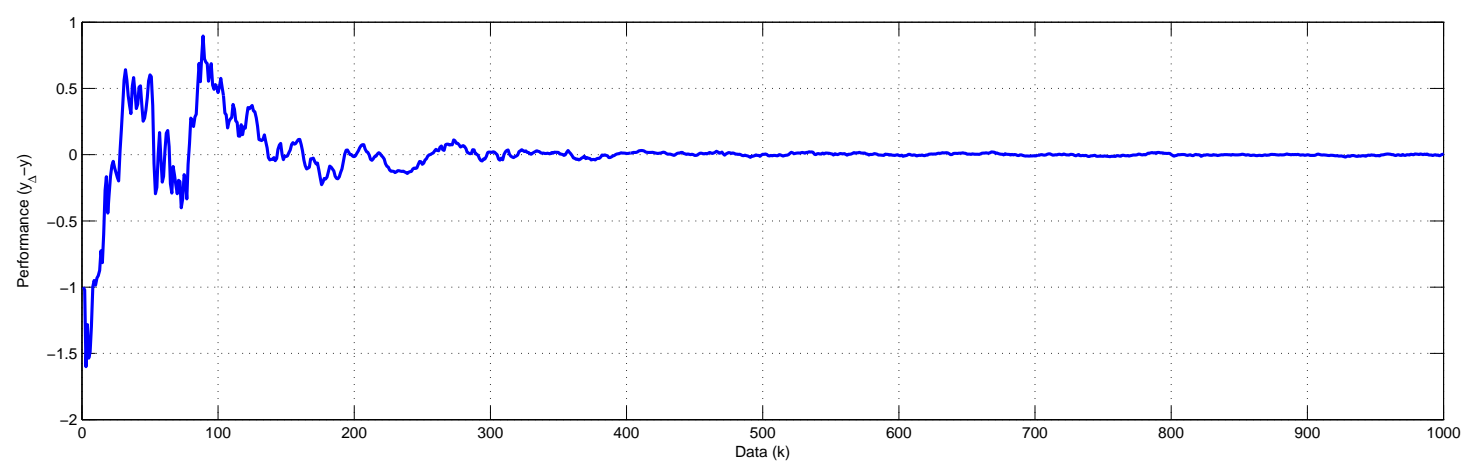

Figure 4. This plot shows the history of the performance variable $y_{\Delta}-y$.

5 compares the state estimation error $x-x_{0}$, without RCO, where $x_{0}$ is the state obtained from the initial model, and $x-x_{\Delta}$, which is the state estimation error with RCO. The state estimation error is reduced using RCO for both states. Using $x_{\Delta}$ obtained in the previous step we set $n_{c}=0, p=100, \mu=1, a=1000$. Setting $n_{c}=0$ results in static estimates of $\hat{\Delta}_{x}$ and $\hat{\Delta}_{w}$. Figure 7 is the performance variable $\hat{y}-y$, using the updated states $x_{\Delta}$. We now consider the case where the state estimation step is omitted, that is, we 


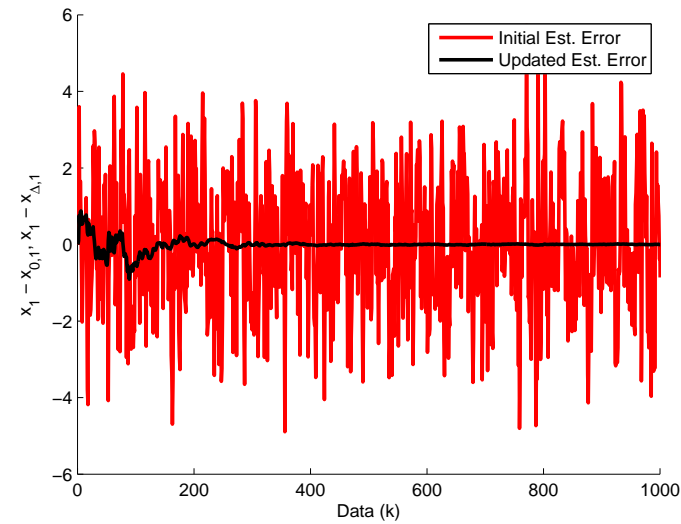

(a)

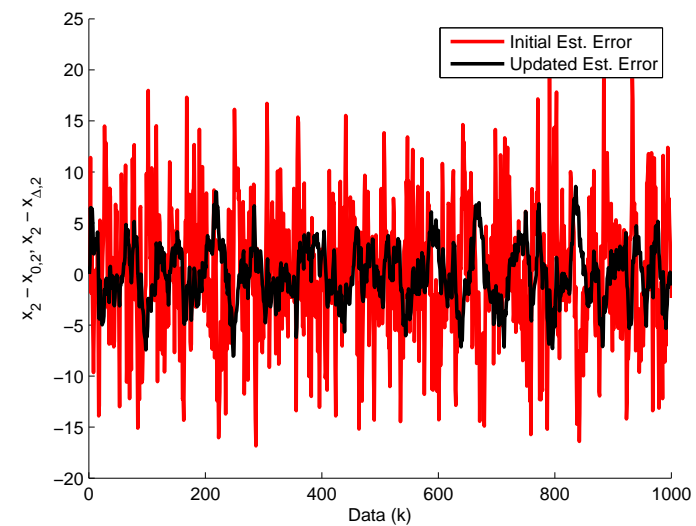

(b)

Figure 5. This plot compares the state estimation error. The red line is $x-x_{0}$, where $x_{0}$ is the state of the initial model. The black line is $x-x_{\Delta}$, where $x_{\Delta}$ is the state of the initial model with $\left[\hat{\Delta}_{x}(\mathbf{q}, k) \hat{\Delta}_{w}(\mathbf{q}, k)\right]$ in feedback.

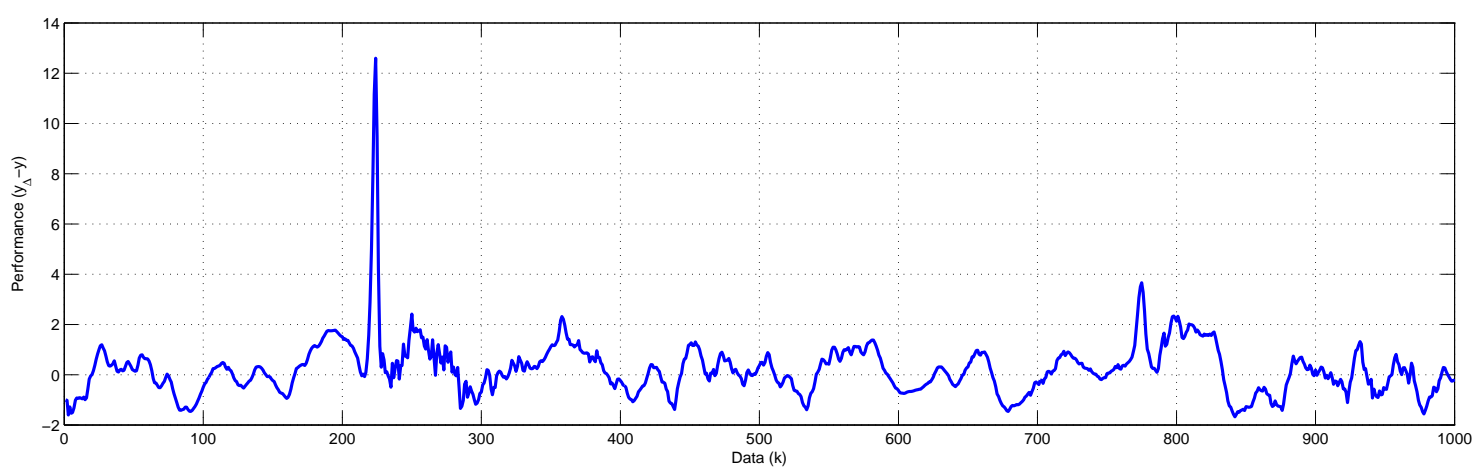

Figure 6. This plot shows the history of the performance variable $\hat{y}-y$, where we set $n_{c}=0$ to obtain $\hat{\Delta}_{x}(k)$ and $\hat{\Delta}_{w}(k)$ without first obtaining state estimates. The resulting parameter estimates are poor.

do not compute $x_{\Delta}$. Figure 6 shows the parameter estimation performance $\hat{y}-y$. The performance in the case that $x_{\Delta}$ is computed results in better performance and, therefore, better parameter estimates. Finally, using $\hat{\Delta}_{x}(k)$ and $\hat{\Delta}_{w}(k)$ we compute estimates of the unknown parameters in the discrete-time case and convert these results to continuous time. Figure 8(a) compares the frequency response of the initial model, the actual system, and the refined model, in discrete time. Figure 8(b) compares the frequency response of the initial model, the actual system, and the refined model, in continuous time. The result is a refined model that better approximates the actual system compared to the initial model.

\section{Damage Localization}

We now present an example where parameter estimation is used for damage localization. Consider the 2-DOF mass-spring-damper system shown in Figure 9. The equations of motion for this system are

$$
M \ddot{x}+C \dot{x}+K x=F
$$




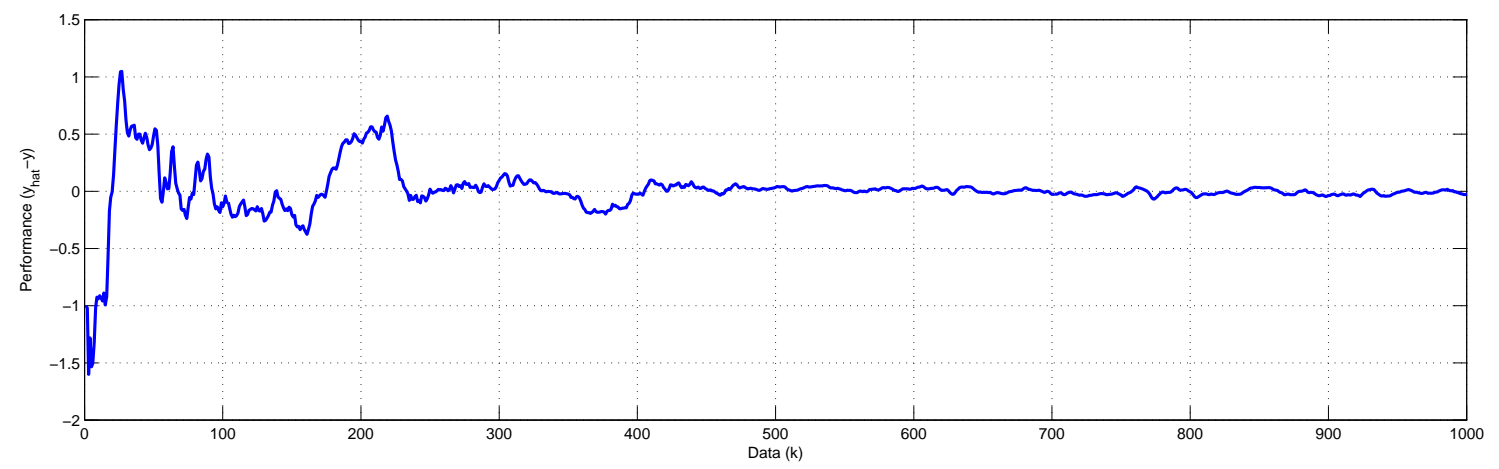

Figure 7. This plot shows the history of the performance variable $\hat{y}-y$, where we set $n_{c}=0$ to obtain $\hat{\Delta}_{x}(k)$ and $\hat{\Delta}_{w}(k)$. In this case we use the state estimate $x_{\Delta}$. The performance is much better than that shown in Figure 6.

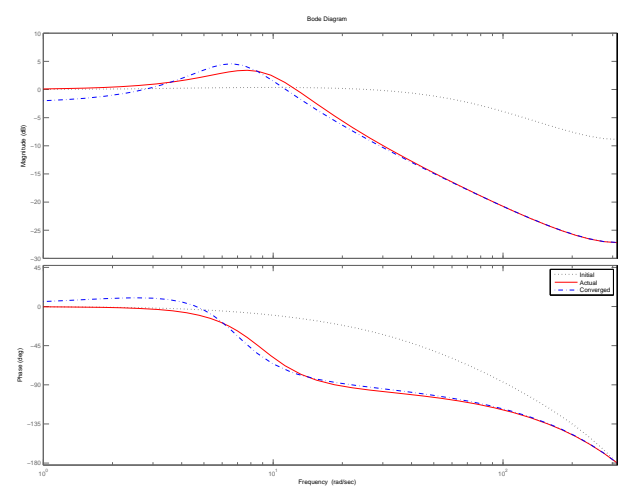

(a)

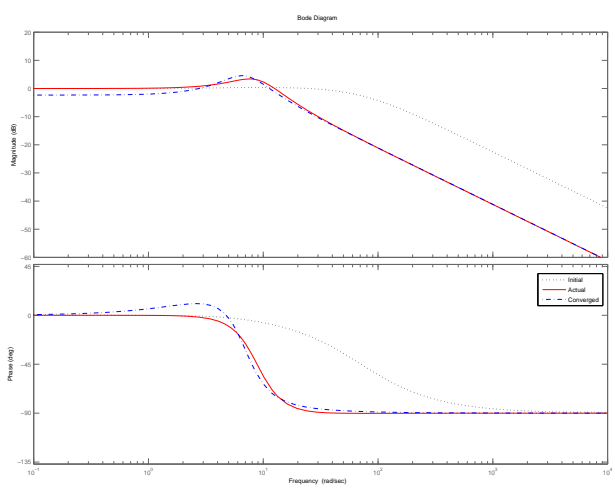

(b)

Figure 8. (a) compares the frequency response of the initial model, the actual system, and the refined model, in discrete time. (b) compares the frequency response of the initial model, the actual system, and the refined model, in continuous time.

where

$$
\begin{aligned}
x & =\left[\begin{array}{l}
q_{1} \\
q_{2}
\end{array}\right], M=\left[\begin{array}{cc}
m_{1} & 0 \\
0 & m_{2}
\end{array}\right], C_{\mathrm{d}}=\left[\begin{array}{cc}
c_{1}+c_{2} & -c_{2} \\
-c_{2} & c_{2}+c_{3}
\end{array}\right], \\
K & =\left[\begin{array}{cc}
k_{1}+k_{2} & -k_{2} \\
-k_{2} & k_{2}+k_{3}
\end{array}\right], F=\left[\begin{array}{l}
f_{1} \\
f_{2}
\end{array}\right] .
\end{aligned}
$$

The mass-spring-damper system can be represented in state space form as

$$
\tilde{A}_{\mathrm{c}}=\left[\begin{array}{cc}
0_{2 \times 2} & I_{2} \\
-M^{-1} K & -M^{-1} C_{\mathrm{d}}
\end{array}\right], \quad \tilde{B}_{\mathrm{c}}=\left[\begin{array}{cc}
0_{2 \times 1} & M^{-1} F
\end{array}\right]^{\mathrm{T}}, \quad \tilde{C}=\left[\begin{array}{cccc}
0 & 0 & 1 & 0
\end{array}\right], \quad D=0 .
$$

In the multi-degree of freedom case the matrices $M, K$, and $C_{\mathrm{d}}$ contain spatial information. That is, these matrices depend on the locations of the mass, stiffness, and damping within the system.

We now consider a system where a change in global stiffness and damping has occurred. Specifically, (50) 


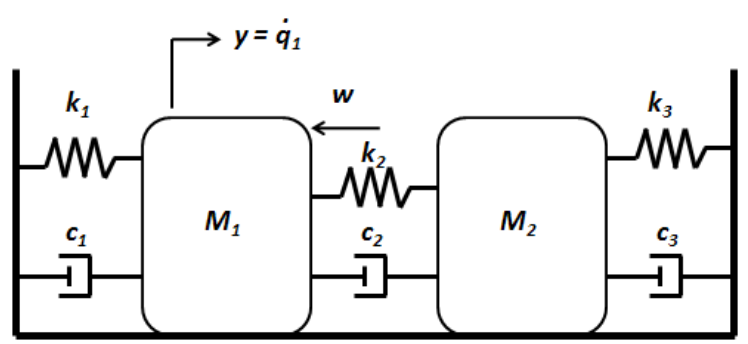

Figure 9. Mass-spring-damper 2-DOF structure.

and (51) become

$$
\dot{x}=\left[\begin{array}{cc}
0_{2 \times 2} & I_{2} \\
M^{-1}(-K+\Delta K) & M^{-1}\left(-C_{\mathrm{d}}+\Delta C_{\mathrm{d}}\right)
\end{array}\right] x+\left[\begin{array}{c}
0_{2 \times 1} \\
M^{-1} F
\end{array}\right] w,
$$

where $\Delta K$ and $\Delta C$ are the changes in stiffness and damping, respectively. The changes to the system can then be written in feedback as

$$
\dot{x}=\left[\begin{array}{cc}
0_{2 \times 2} & I_{2} \\
-M^{-1} K & -M^{-1} C_{\mathrm{d}}
\end{array}\right] x+\left[\begin{array}{cc}
0_{2 \times 2} & 0_{2 \times 2} \\
0_{2 \times 2} & M^{-1}
\end{array}\right] u+\left[\begin{array}{c}
0_{2 \times 1} \\
F
\end{array}\right] w .
$$

where

$$
u=\left[\begin{array}{cc}
0_{2 \times 2} & 0_{2 \times 2} \\
\Delta K & \Delta C_{\mathrm{d}}
\end{array}\right] x .
$$

We choose $m_{1}=0.8, m_{2}=0.5, c_{1}=100, c_{2}=200, c_{3}=120, k_{1}=1000, k_{2}=25000, k_{3}=25000, f_{1}=1000$, $f_{2}=0$, which are the parameters of the initial model. Furthermore, assume that the physical system has undergone a change such that $k_{2}+\Delta k_{2}=1500$, and $k_{3}+\Delta k_{3}=2000$.

We implement RCO to obtain estimates of the transfer functions $\hat{\Delta}_{x}(\mathbf{q}, k)$ and $\hat{\Delta}_{w}(\mathbf{q}, k)$, which are were used to obtain $x_{\Delta}$. The RCO parameter selections are $n_{c}=5, p=100, \mu=1, a=100$. Figure 4 shows the history of the performance variable $y_{\Delta}-y$. Figure 11 compares the state estimation error $x-x_{0}$ without

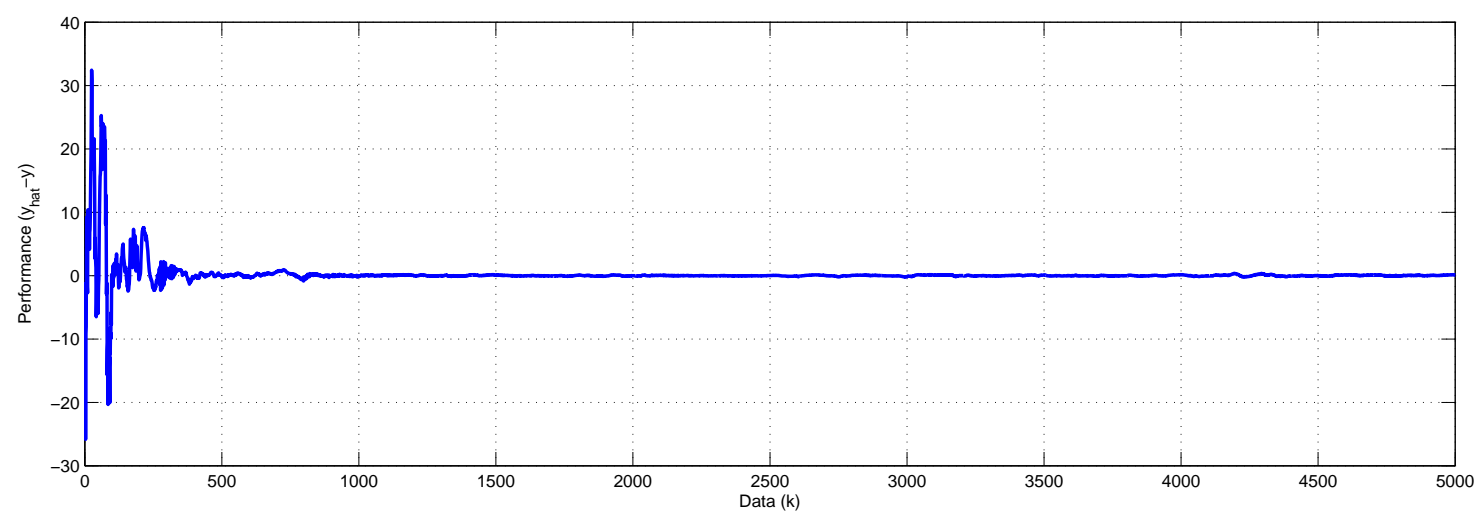

Figure 10. This plot shows the history of the performance variable $y_{\Delta}-y$ for the 2-DOF system.

$\mathrm{RCO}$, where $x_{0}$ is the state obtained from the initial model, and state estimation error, $x-x_{\Delta}$, with $\mathrm{RCO}$. 


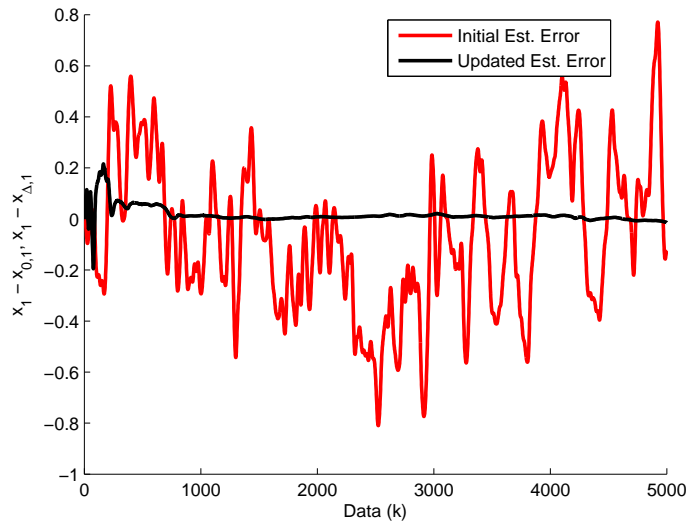

(a)

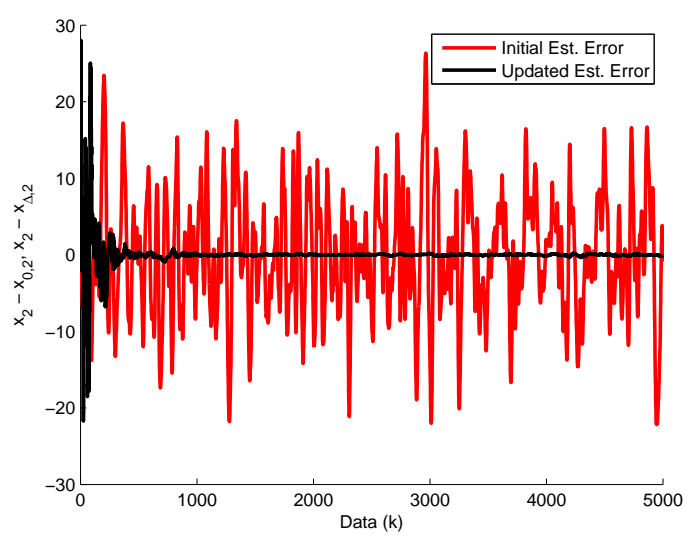

(c)

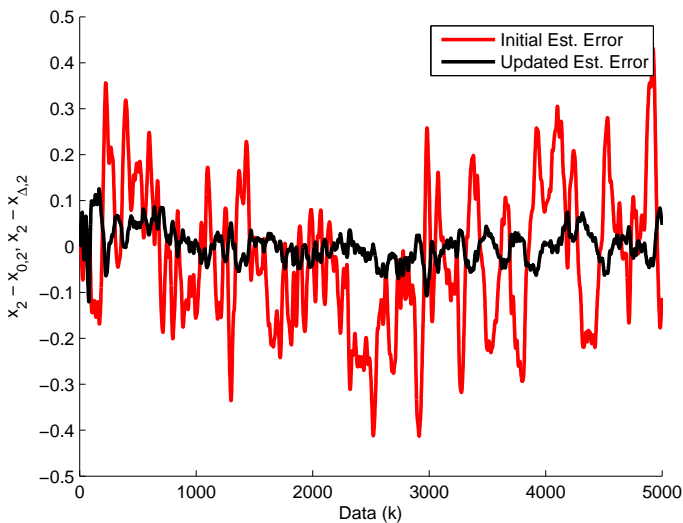

(b)

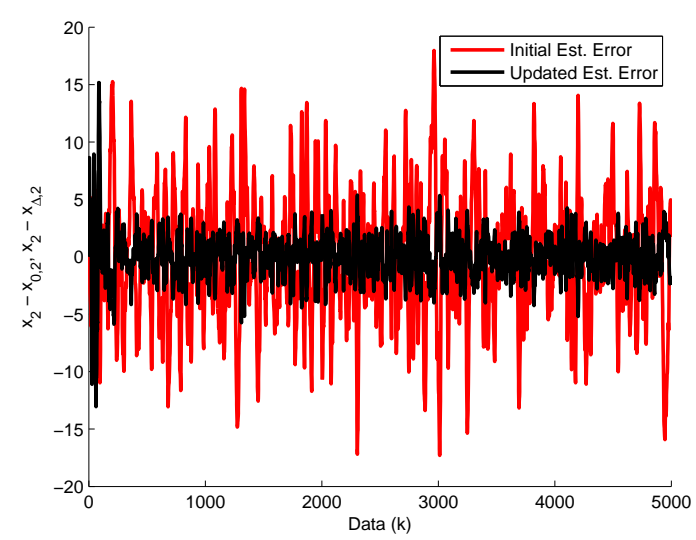

(d)

Figure 11. This plot compares the state estimation error. The red line is $x-x_{0}$, where $x_{0}$ is the state of the initial model. The black line is $x-x_{\Delta}$, where $x_{\Delta}$ is the state of the initial model with $\left[\hat{\Delta}_{x}(\mathbf{q}, k) \hat{\Delta}_{w}(\mathbf{q}, k)\right]$ in feedback.

The state estimation error is reduced for all 4 states. Using $x_{\Delta}$ obtained in the previous step, we set $n_{c}=0$, $p=100, \mu=1, a=1000$. Figure 12 shows the performance variable $\hat{y}-y$, using the updated states $x_{\Delta}$.

Finally, using $\hat{\Delta}_{x}(k)$ and $\hat{\Delta}_{w}(k)$ we compute estimates of the unknown parameters in the discrete-time case, and convert these results to continuous time. Figure 13(a) compares the frequency response of the initial model, the actual system, and the refined model, in discrete time. Figure 13(b) compares the frequency response of the initial model, the actual system, and the refined model, in continuous time. The result is a refined model that better approximates the actual system when compared to the initial model.

In the multi-degree of freedom case, refining the initial model where mass and damping are assumed to be known, estimates of the refined stiffness matrix provides quantification of the change in stiffness as well as the location in the model.

\section{Experimental Results}

To demonstrate the model refinement method experimentally, we construct a series resistor-inductorcapacitor (RLC) circuit, which is analogous to the mass-spring-damper system. Consider the RLC circuit shown in Figure 14 modeled by

$$
L \ddot{x}+R \dot{x}+\frac{1}{C_{\mathrm{d}}} x=u
$$

where $L, C_{\mathrm{d}}, K$ are the inductor, capacitor, and resistor values, respectively, and $w$ is the input voltage. We 


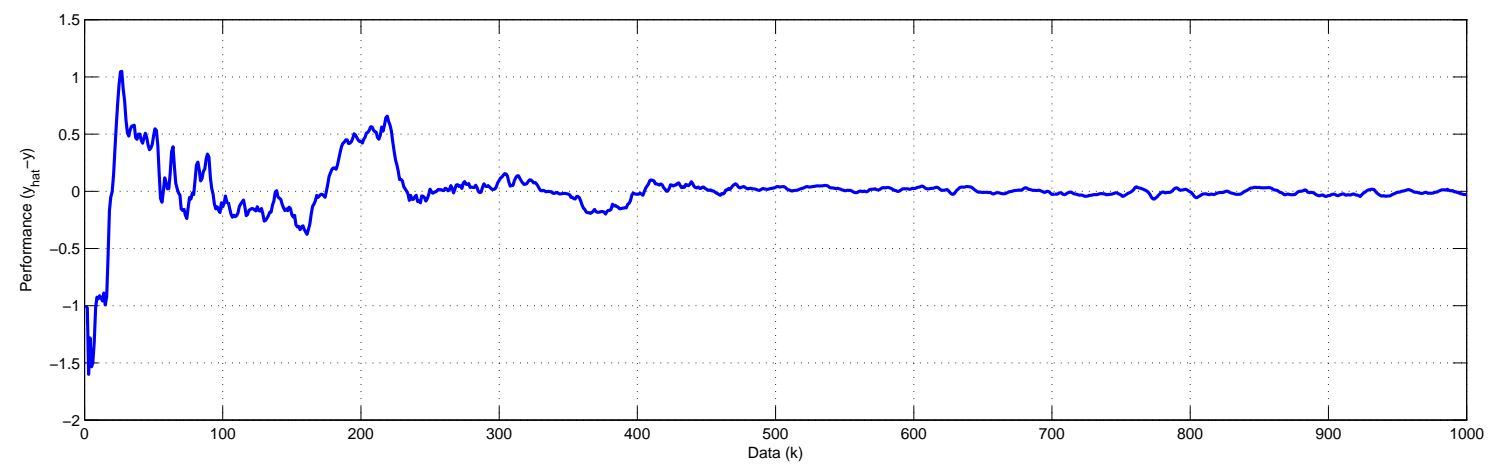

Figure 12. This plot shows the history of the performance variable $\hat{y}-y$, where we set $n_{c}=0$ to obtain $\hat{\Delta}_{x}(k)$ and $\hat{\Delta}_{w}(k)$. In this case we use the state estimate $x_{\Delta}$ to update constant parameters $\left[\hat{\Delta}_{x} \hat{\Delta}_{w}\right]$

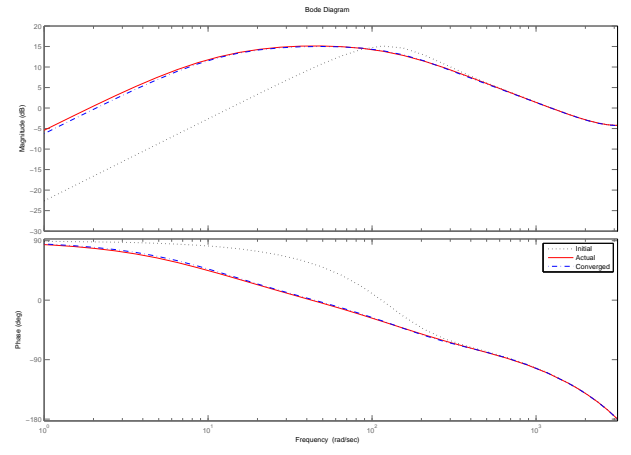

(a)

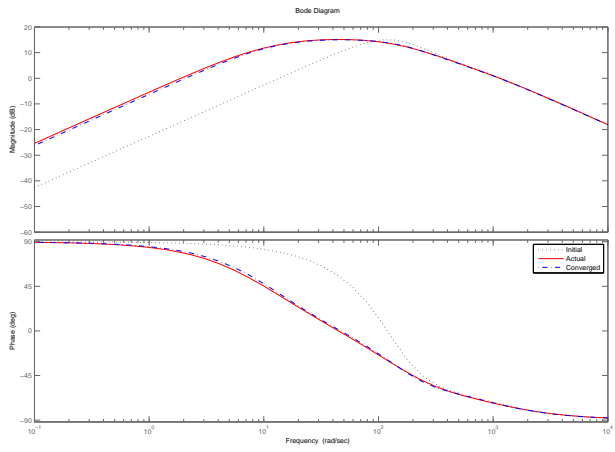

(b)

Figure 13. (a) compares the frequency response of the initial model, the actual system, and the refined model, in discrete time. (b) compares the frequency response of the initial model, the actual system, and the refined model, in continuous time.

obtain the state space representation of the circuit

$$
\begin{aligned}
{\left[\begin{array}{c}
\dot{x} \\
\ddot{x}
\end{array}\right] } & =\left[\begin{array}{cc}
0 & 1 \\
-\frac{1}{L C_{\mathrm{d}}} & -\frac{R}{L}
\end{array}\right]\left[\begin{array}{l}
x \\
\dot{x}
\end{array}\right]+\left[\begin{array}{c}
0 \\
\frac{1}{L}
\end{array}\right] u, \\
y & =\left[\begin{array}{ll}
0 & R
\end{array}\right]\left[\begin{array}{l}
x \\
\dot{x}
\end{array}\right],
\end{aligned}
$$

where $x$ and $\dot{x}$ are the charge and current, respectively, of the circuit. Furthermore, we write the state space equations for a circuit with an unknown change in capacitance $\Delta C_{\mathrm{d}}$ and inductance $\Delta L$ as

$$
\begin{aligned}
{\left[\begin{array}{l}
\dot{x} \\
\ddot{x}
\end{array}\right] } & =\left[\begin{array}{cc}
0 & 1 \\
-\frac{1}{(L+\Delta L)\left(C_{\mathrm{d}}+\Delta C_{\mathrm{d}}\right)} & -\frac{R}{L+\Delta L}
\end{array}\right]\left[\begin{array}{l}
x \\
\dot{x}
\end{array}\right]+\left[\begin{array}{c}
0 \\
\frac{1}{L+\Delta L}
\end{array}\right] w, \\
y & =\left[\begin{array}{ll}
0 & R
\end{array}\right]\left[\begin{array}{l}
x \\
\dot{x}
\end{array}\right],
\end{aligned}
$$




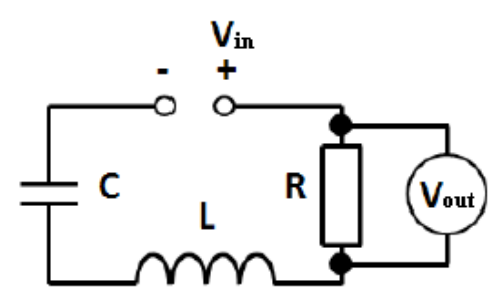

Figure 14. A series resistor-inductor-capacitor (RLC) circuit, where voltage is measured across the resistor.

where $u=\Delta_{x} x+\Delta_{\dot{x}} \dot{x}+\Delta_{w} w$. We can then compute an estimate $\Delta \hat{C}_{\mathrm{d}}$ of $\Delta C_{\mathrm{d}}$ and an estimate $\Delta \hat{L}$ of $\Delta L$ from the converged adaptive controller by using

$$
\begin{aligned}
\Delta \hat{L} & =\frac{L}{\Delta_{w}}-L=\frac{-R L}{-R+\Delta_{\dot{x}}}-L, \\
\Delta \hat{C}_{\mathrm{d}} & =-L\left(\frac{-1}{C_{\mathrm{d}}}+\Delta_{x}\right)^{-1}(L+\Delta L)^{-1}-C_{\mathrm{d}} .
\end{aligned}
$$

We assemble a circuit with $R=250 \Omega, L+\Delta L=55 \mathrm{mH}$, and $C_{\mathrm{d}}+\Delta C_{\mathrm{d}}=23.5 \mu \mathrm{F}$. We assume that we do not have knowledge of $\Delta C_{\mathrm{d}}$ or $\Delta L$, but we estimate $C_{\mathrm{d}}=1 \mathrm{~F}$ and $L=2 \mu \mathrm{H}$. The model (57) is discretized using a zero order hold. We drive the circuit using a zero-mean, Gaussian white noise, and we measure the voltage across the resistor. The driving signal and measurement are recorded using a DSPACE setup.

We implement RCO to obtain estimates of the transfer functions $\hat{\Delta}_{x}(\mathbf{q}, k)$ and $\hat{\Delta}_{w}(\mathbf{q}, k)$ used to obtain $x_{\Delta}$. The RCO parameter selections are $n_{c}=5, p=100, \mu=1, a=100$. Figure 15 shows the history of the performance variable $y_{\Delta}-y$. Figure 16 shows a comparison between the state estimation error without

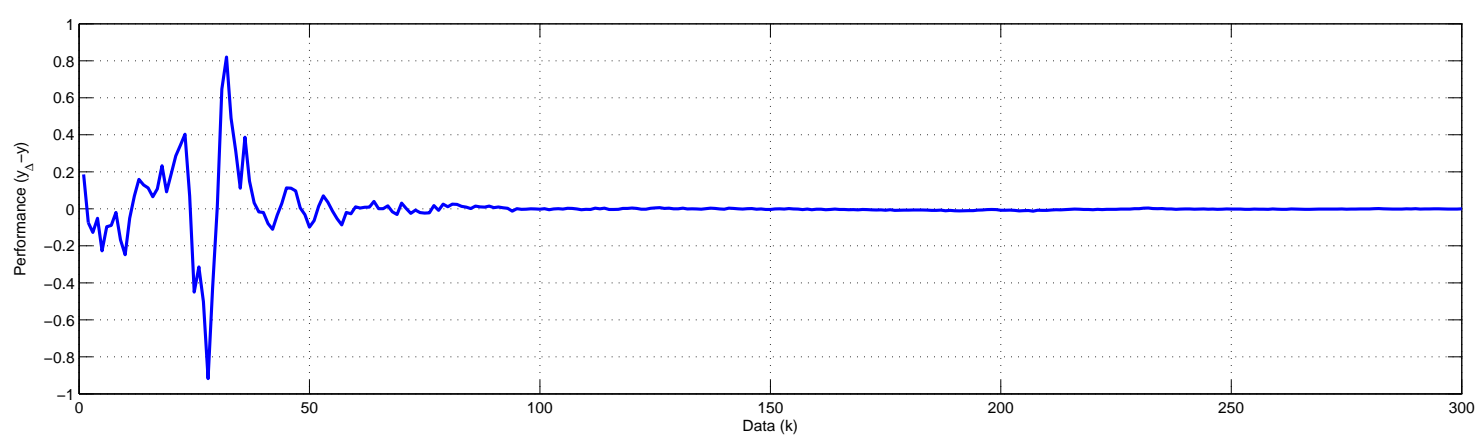

Figure 15. This plot shows the history of the performance variable $y_{\Delta}-y$.

RCO, $x-x_{0}$, where $x_{0}$ is the state obtained from the initial model, and $x-x_{\Delta}$ which is the state estimation error with RCO. Figure 17 is a comparison between the state estimation error $x-x_{0}$, without RCO, where $x_{0}$ is the state obtained from the initial model, and the state estimation error $x-x_{\Delta}$, when RCO is used. Finally, using $\hat{\Delta}_{x}(k)$ and $\hat{\Delta}_{w}(k)$ we compute estimates of the unknown parameters in the discrete time case, and convert these results to continuous-time. Figure 18(a) is a comparison of the frequency response of the initial model, the actual system, and the refined model, in discrete-time. Figure 18(b) is a comparison of the frequency response of the initial model, the actual system, and the refined model, in continuous-time. The result is a refined model which better approximates the actual system compared to the initial model. 


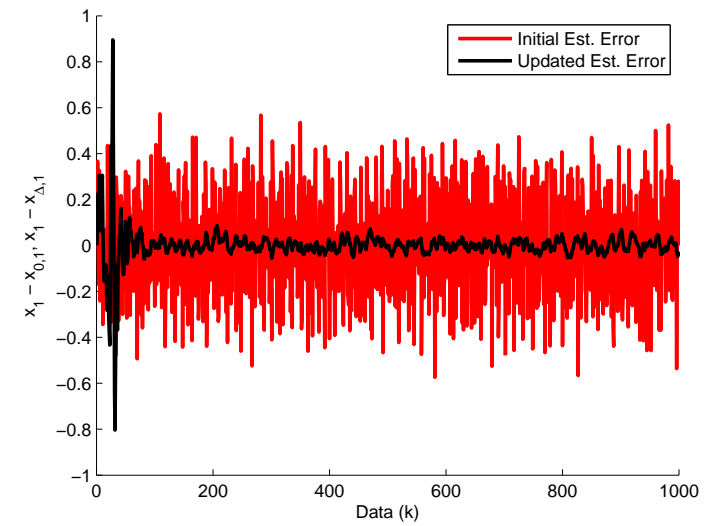

(a)

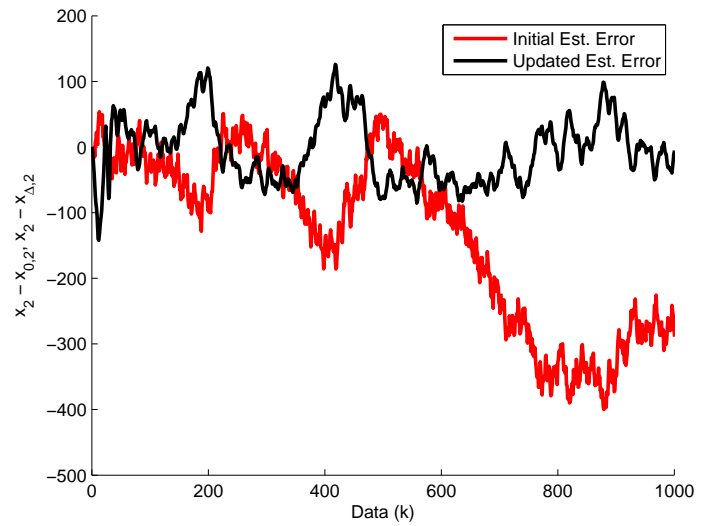

(b)

Figure 16. This plot compares the state estimation error. The red line is $x-x_{0}$, where $x_{0}$ is the state of the initial model. The black line is $x-x_{\Delta}$, where $x_{\Delta}$ is the state of the initial model with $\left[\hat{\Delta}_{x}(\mathbf{q}, k) \hat{\Delta}_{w}(\mathbf{q}, k)\right]$ in feedback.

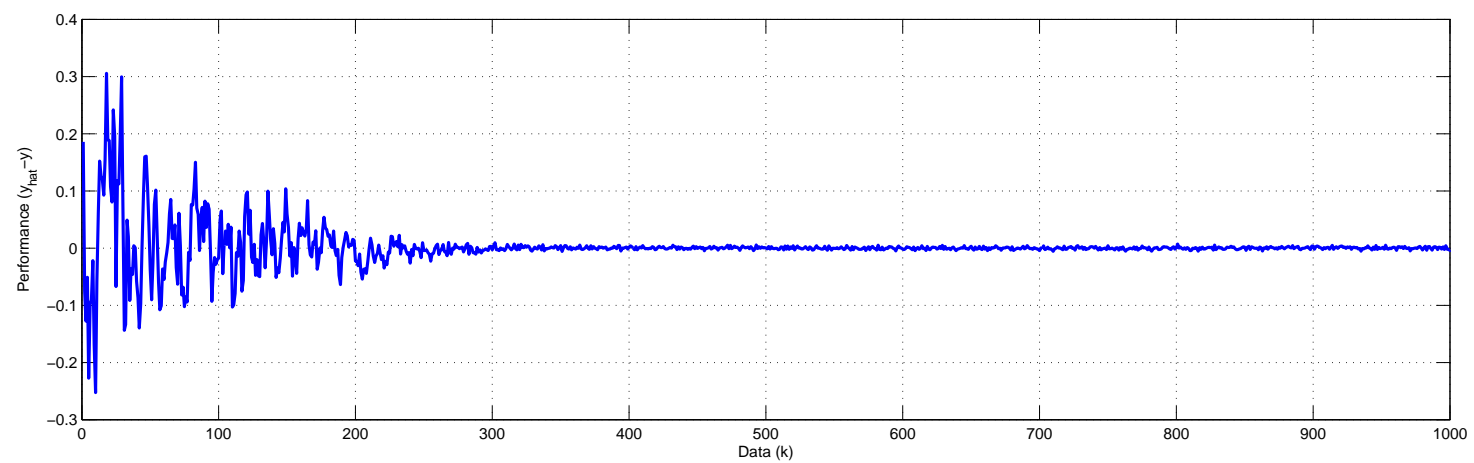

Figure 17. This plot shows the history of the performance variable $\hat{y}-y$, where we set $n_{c}=0$ to obtain $\hat{\Delta}_{x}(k)$ and $\hat{\Delta}_{w}(k)$. In this case we use the state estimate $x_{\Delta}$.

\section{Conclusion}

In previous work, model refinement was used within the scope of structural health monitoring to detect global damage in a structural member. Furthermore, it was demonstrated that system models could be updated using model refinement techniques to reflect the changes in system dynamics due to the global damage.

We now apply model refinement techniques with an assumed model structure, for example, a mass-springdamper system whose damage can be characterized in terms of changes in stiffness and damping and also localized spatially in the model. We use a fixed initial model with retrospective cost optimization to obtain state estimates of the physical system in the presence of an unknown system model. The refined model obtained during the state estimation step is based on a dynamic update and therefore does not provide accurate estimates of the unknown parameters. Using the updated state estimates, a static correction is estimated which in turn is used to determine new estimates of the unknown parameters. We demonstrated this concept on a single degree of freedom application, where change to the stiffness and damping are determined. Furthermore, we applied the method to a 2 degree of freedom system, which had undergone changes to the global stiffness. In this example, corrections to the stiffness matrix also provide localization of the changes to the system, since the global stiffness matrix depends on spatial locations of the springs within the structure. Finally, experimental data from an RLC circuit was used to demonstrate the capability 


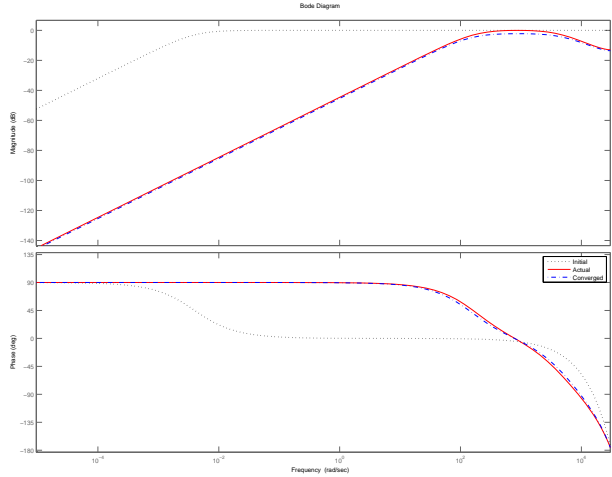

(a)

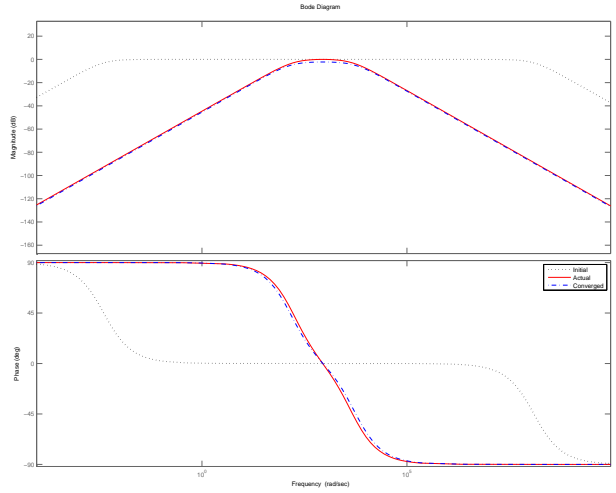

(b)

Figure 18. (a) compares the frequency response of the initial model, the actual system, and the refined model, in discrete time. (b) compares the frequency response of the initial model, the actual system, and the refined model, in continuous time.

to determine changes in capacitance and inductance. This example is the analogue to the single degree of freedom mass-spring-damper system.

\section{References}

${ }^{1}$ C. Minas and D. Inman. Matching finite element models to modal data. J. Vibration Acoust., vol. 112, pp. 84-92, 1990.

${ }^{2}$ J.B. Carvalho, B. N. Datta, W. Lin, and C. Wang. Symmetry preserving eigenvalue embedding in finite-element model updating of vibrating structures. J. Sound Vibration, 290 (2006) 839-864.

${ }^{3}$ M. I. Friswell and J. E. Mottershead. Finite Element Model Updating in Structural Dynamics. Kluwer, Dordrecht, 1995.

${ }^{4}$ F. D'Andrea and R. Vautard. Reducing systematic errors by empirically correcting model errors. Tellus, vol. 52A, pp. $21-41,2000$.

${ }^{5}$ T. DelSole and A. Y. Hou. Empirical correction of a dynamical model. Part I: Fundamental issues. Monthly Weather Rev., vol. 127(11), pp. 2533-2545, 2001.

${ }^{6}$ C. M. Danforth, E. Kalnay, and T. Miyoshi. Estimating and correcting global weather model error. Monthly Weather Rev., vol. 135(2), pp. 281-299, 2007.

${ }^{7}$ S. O. R. Moheimani. Model correction for sampled-data models of structures. J. Guidance, Contr., and Dynamics, vol. 24(3), pp. 634-637, 2001.

${ }^{8}$ S. Mijanovic, G. E. Stewart, G. A. Dumont, and M. S. Davies. A controller perturbation technique for transferring closed-loop stability between systems, Automatica, vol. 39, pp. 1783-1791, 2003.

${ }^{9}$ H. Palanthandalam-Madapusi, E. L. Renk, and D. S. Bernstein. Data-Based Model Refinement for Linear and Hammerstein Systems Using Subspace Identification and Adaptive Disturbance Rejection. Proc. Conf. Contr. Appl., pp. 1630-1635, Toronto, Canada, August 2005.

${ }^{10}$ M. A. Santillo, A. M. D'Amato, and D. S. Bernstein, "System Identification Using a Retrospective Correction Filter for Adaptive Feedback Model Updating," Proc. Amer. Contr. Conf., pp. 4392-4397, St. Louis, MO, June 2009.

${ }^{11}$ A. M. D'Amato and D. S. Bernstein, "Linear Fractional Transformation Identification Using Retrospective Cost Optimization," Proc. SYSID, pp. 450-455, Saint-Malo, France, July 2009.

${ }^{12}$ A. M. D'Amato, B. J. Arritt, J. A. Banik, E. V. Ardelean, and D. S. Bernstein, "Structural Health Determination and Model Refinement for a Deployable Composite Boom," AIA A SDM Conf., Palm Springs, CA, April 2009, AIAA-2009-2373.

${ }^{13}$ M. A. Santillo and D. S. Bernstein, "Adaptive Control Based on Retrospective Cost Optimization," AIAA J. Guid. Contr. Dyn., Vol. 33, pp. 289-304, 2010.

${ }^{14}$ J. Barhen and V. Protopopescu and D. B. Reister, "Consistent uncertainty reduction in modeling nonlinear systems," SIAM J. Sci. Comput., Vol. 26, pp. 653-665, 2004. 